\title{
Paraplegia
}

\section{Review Article: The Amelioration and Cure of Spinal Cord Damage. The Aim of the International Spinal Research Trust}

\author{
P. J. Banyard, MA, ${ }^{1}$ L. S. Illis, FRCP, ${ }^{2}$ P. D. Wall, FRS, FRCP ${ }^{3}$ \\ ${ }^{1}$ International Spinal Research Trust, Nicholas House, Middlesex, ${ }^{2}$ Wessex Neuro- \\ logical Centre, Southampton General Hospital, ${ }^{3}$ Department of Anatomy and Develop- \\ mental Biology, University College London, UK.
}

The International Spinal Research Trust (ISRT) was founded in 1980 with the aim of helping to encourage an international effort directed toward the amelioration and cure of spinal cord damage. Its founder, Stewart Yesner, was himself quadriplegic, which meant that ISRT was an expression of the wishes of those paralysed by injury or disease. Because of this, and because fraud and charlatanry are not absolute strangers to the field, it was carefully structured. Yesner was fortunate in securing the voluntary services of an eminently qualified Scientific Committee. Its members have given critical advice to the Trustees over the years and ensured that the projects supported have been both scientifically respectable and correctly targeted at the problems facing repair.

Millions of pounds sterling have been collected and disbursed since 1980, and they have been used for two purposes: education and research. Education has been fostered by meetings, by the publication of a Medical Research Intelligence Summary which collates all relevant work undertaken in international laboratories, and by the publication so far of three books on spinal dysfunction, assessment and treatment. However, the largest effort is spent on the support of clinical and basic research. The reasons and need for this we now summarise.

\section{Basic studies}

We are living through an extraordinarily exciting scientific period in which the utter pessimism about the possibility of any form of cure for paraplegia or any other form of central nervous system damage is being replaced very rapidly by guarded optimism. The pessimism was fully justified by the repeated observation of the complete failure of regeneration in any mammallian adult brain or spinal cord. The guarded optimism comes from two sources, first specific discoveries and second a general methodology. An example of specific discovery comes from Aguayo's group in Montreal who showed that if cut adult central axons were placed in contact with Schwann cells they regenerated over very long distances. At a 
stroke, a supposed fact on which the old pessimism was based, was abolished. It had been imagined that some factors within adult central cells prohibited the regeneration of their axons. The revolution implicit in Aguayo's discovery was that the fault lay not entirely in the nerve cells but in the cells they contacted. The exploitation of this new fact shows the extraordinary power of the second factor, molecular biology, to discover, analyse and manipulate sources of prohibition and of encouragement of regeneration. A brilliant example is the work of Schwab's group in Zurich who first defined precisely the molecules on the surface of oligodendrocytes which inhibited the growth of axons in tissue culture. Then they made antibodies to those molecules to neutralise their effect. Next in order to generate a living source of these antibodies, they engineered neuroblastoma cells to produce the antibodies and transplanted them into rats with partial cord sections. The result was a clear elongation of cut axons through the region of damage. This remarkable series of experiments is quoted not because it produces a definitive cure but because it shows the power and speed of the new techniques.

It now becomes possible not only to analyse precisely why regeneration does not occur but to define a set of plausible achievable strategies by which the prohibitions might be overcome. The answer is not going to depend on a single factor but on the recognition of a sequence of interacting changes which follow injury. When a nerve cell has its own axon cut, it is isolated from the normal end station which supplies a continuous chemical signal which informs the cell that the axon is in contact with its 'correct' target. In the absence of this continual reassurance, the cell becomes sickly and may die. Levi-Montalcini and her colleagues won their Nobel prize for their identification of one such signal molecule, nerve growth factor, which is made in the periphery. Now a massive search is on, with early signs of success, for equivalent brain growth factors. There is no doubt that there is something wrong with adult nerve cells since perhaps the most important result of transplantation experiments is to show that fetal tissue transplanted into adult brain can still grow and sometimes find its correct target. This fact challenges the new generation of scientist to define precisely what substances permit this fetal ability and to consider how they might be reformed in adult cells. Once sprouts have been encouraged to grow from a cut axon, there is no doubt that they collide with adult inhibitory factors. We have already mentioned oligodendrocytes as one source but it is certain that there are others including myelin and above all the astrocytes. It is clear that there are good and bad astrocytes. What constitutes their goodness and badness? Could the bad ones be reformed? Could the good ones be introduced? These are no longer science fiction fantasies but the subject of a growing research effort by very hard headed and bright scientists with powerful new tools.

In parallel with these attempts to restore destroyed connections there is the equally crucial practical question of how the surviving nervous system reacts to damage of neighbouring cells. Just as the possibility of regeneration is now revealed, it now becomes apparent that the old idea of a rigid fixed hard wired adult nervous system was equally wrong. Adult synaptic contacts are not simply soldered in place during embryonic development to remain stationary throughout adult life. Activity or lack of activity controls the effectiveness of large numbers of synapses. Intact nerves sense the presence of nearby degeneration and sprout to occupy the vacant sites. Ineffective synapses become effective partly driven by the presence of nerve impulse activity and partly by trophic factors. This plasticity of 
connection in the adult nervous system contains good and bad news. The bad news is that inappropriate novel connections may form in the presence of partial damage and any recovery will necessitate the prevention or removal of these new connections. The good news is that, in the presence of the very common partial cord lesions, it may be possible to encourage novel appropriate and beneficial connections by taking advantage of the remaining plasticity of the existing adult nerve cells and their axons.

\section{Clinical studies}

The idea of cure in spinal injury suggests an all or nothing approach which is appealing but not immediately realistic. Restoring function, on the other hand, more accurately describes current research and suggests that there may be several steps, any one of which may improve lifestyle, reduce degree of dependence and alleviate distressing symptoms. There are three main approaches to restoration of function:

\section{The prevention of secondary pathological events following spinal cord injury}

The early events found in animal models of spinal cord damage indicate evidence of progressive tissue damage. Metabolic disturbances, the release or activation of toxic substances, ionic shifts and a fall in blood flow in the white matter produce damage at the lesion site. Calcium entry into cells affects mitochondrial transport and initiates lipid peroxidation which, in turn, releases free radicals causing a continuing injury response resulting in progressive membrane damage, oedema and a further fall in blood flow.

Several different types of drug treatment have been used in spinal cord injury (SCI) and although blood flow improvement has been demonstrated in experimental studies, and some functional recovery has been reported, the results are inconsistent. The effects appear to vary with the type of model used, the drug dosage and the timing of the drug treatment. The drugs studies include Naloxone, Thyrotrophin-releasing hormone, opiate receptor blockers, 21-amino steroids, Aminophylline and Methylprednisolone. In human SCI the use of Methylprednisolone appears to be the most favourable. A recent randomised, placebocontrolled, double-blind trial carried out in ten centres in the USA studied 162 patients treated with intravenous Methylprednisolone, 171 patients with placebo, and 154 patients treated with Naloxone. Naloxone showed no effect, but patients treated with high-dose Methylprednisolone showed significant improvements in motor and sensory function compared to placebo. This improvement was noted at 6 weeks and 6 months after the injury. However, the improvement was only seen in those patients who started treatment within 8 hours of the injury and emphasizes the importance of early treatment and also the importance of trying to delineate precisely the natural history of SCI within the first few hours. Perhaps even better results could be obtained using intrathecal drug delivery systems, and (in the future), using antibodies to growth inhibitory factors. After severe trauma to the spinal cord, pericapillary haemorrhages occur in the grey matter, followed, after an interval, by central haemorrhagic necrosis of the cord with involvement of the white matter at the later stage. Most of the cord damage has occurred within the 
first 8 hours so that again early treatment is essential. Local spinal cord cooling has been used experimentally and in Man. Spinal cord blood flow can be measured. In Man local cooling together with steroids has shown benefit, possibly by preventing the occurrence of oedema or by a reduction in tissue metabolism and the release of toxic substances mentioned above.

A potassium channel blocker, 4-aminopyridine, can restore conduction in damaged (demyelinated) fibres and may improve recovery where conduction block is a factor. This drug has been used in experimental models of SCI, and a trial in Man is underway.

2. Identification of impaired or absent function in nerve cells and processes which survive the initial insult intact but with impaired function

The lesion itself is not wholly responsible for the observed methodological deficit. Part of the deficit is due to altered function in the intact nervous system and if we knew more about the way the intact nervous system reacts then it might be possible to influence recovery. Some of the secondary alteration of function may be prevented by the methods described above or by the use of stimulation techniques which alter inhibition.

Stimulation techniques fall largely into two groups: those which are thought to alter CNS function (probably by altering inhibition) and those which involve direct stimulation of nerve or muscle, and are designed to reproduce the functions lost as a result of the central lesion.

The first group includes spinal cord stimulation. In this technique electrodes are placed in the epidural space to give a continuous stimulation adjusted by the patient to give a pleasant warm tingling sensation. This technique has been used to treat the effects of spinal lesions, and stems directly from the anatomical and physiological studies which formed the basis of the 'gate-control' theory of pain. It has been in use for some 20 years and has shown benefit in spasticity, pain and bladder disturbance, with concomitant alteration in physiological parameters. In spinal injury patients it may produce improvement in spasticity in about two thirds of patients.

The most effective example of the second group is direct sacral root stimulation which has been shown to be a worthwhile method for controlling the bladder in patients with spinal cord injury. Functional electrical stimulation techniques such as lateral popliteal nerve stimulation with an electrode over the lateral popliteal nerve, triggered by a foot switch, may be extremely effective in foot drop due to a central lesion and with an intact peripheral nervous system. One of the largest fields of direct stimulation (in terms of time involved and money spent) is multisite stimulation of muscles and/or peripheral nerves in an attempt to restore motor function. It is a technique which does, indeed, work. Patients with SCI have, with this elaborate technique, stood, cycled, and walked a few paces. However, as a therapeutic programme for the patient, it has not so far shown any widespread application. Perhaps it's major advantage is as an exercise method, improving muscle tone, peripheral circulation and bone metabolism rather than altering neurological function and disability.

\section{Restoration of neuronal connections severed at the time of spinal cord injury}

One of the classical approaches for studying the possibility of regeneration of 
central fibres is to produce a partial or total transection of the spinal cord in an experimental animal and then insert some kind of bridge which will aid or stimulate reconnection of severed axons. The bridges (implants) which have been used include fetal cells, pieces of peripheral nerves, pieces of salivary gland and collagen. The combination of collage matrix and a pedicle omentum graft has been reported to produce an increase in spinal cord blood-flow and regeneration of axons but this work needs to be confirmed. No one knows that the sectioned spinal cord has an inadequate blood supply, (as opposed to a fall in blood flow) and the indentification of the source of the apparently regenerating axons remains controversial.

What does the future look like? Firstly, it must be emphasized that the priority for clinicians is to delineate the natural history of SCI as accurately as possible, particularly the first few hours after SCI. This must involve the use of physiological techniques to measure the integrity or otherwise of nerve pathways. Most of the techniques necessary are already available but very little used (for example, the use of magnetic brain stimulation to assess motor pathways). Methods for assessing spinal cord bloodflow and metabolic measurements need to be developed and these must be correlated with the clinical findings. The effectiveness of present and future therapies can only be anecdotal and controversial unless a rigorous natural history is established by the spinal injury centres. Stimulation techniques will continue to be useful but limited. Multi-site stimulation with impressive computer control will continue to attract vast research money but is unlikely to produce a great deal of patient benefit. Drug trials, such as the use of Methylprednisolone, will continue as a fruitful research field and, in the future, may include nerve growth factors or antibodies against neurite growth inhibitors, introduced by intrathecal catheter to the injury sites. Assuming that severed neuronal connections can be restored by these techniques to the chronically injured spinal cord, this would lead to the even greater problem of target recognition. Faulty connection may lead to a deterioration in function (e.g. spasticity or pain) even though biological restoration is otherwise a success. In spinal injury there is no evidence for an intrinsically abnormal reflex pattern. The abnormality is of heightened excitability and deficient supraspinal control of spinal cord circuitry. In lower vertebrates a spinal pattern generator exists. That is, the essential pattern of coordinated activity responsible for locomotion arises from central neural mechanisms-the central pattern generator (CPG). In the cat and in other lower vertebrates, the CPG can act soon after injury and stepping movements become progressively stronger indicating (a) the integrity of the CPG and (b) the presence of peripheral mechanisms which provide sensory feedback and cause adaptive changes in timing and amplitude of the CPG rhythm. No such organisation has been demonstrated in Man. If such a mechanism exists then it is presumed to be heavily inhibited. If so, then 'regeneration therapy' may be abortive from a functional point of view even if it is a histological success.

In summary, the early treatment of SCI may prevent secondary pathological events including the progression of vascular changes and the destructive effects of free radicals. Impaired or absent function of otherwise intact nerve cells and processes need to be identified. Regeneration of neuronal tissue becomes an increasing and exciting possibility from a biological point of view but may lead to further problems. Despite this, it is essential that the work should be pursued. 


\section{Conclusion}

It is not reasonable to doubt that a cure for paralysis will be found one day. The Trust's function is to ensure that day comes soon rather than late. There is an enormous amount of work to be done and absolutely no prospect that all the necessary funds can be found from the resources of one charity: however large it grows. Despite this, the very existence of the Trust can be a potent focus for the realisation of its aims if its work is carefully directed.

Of course there is a need for a greatly expanded funding budget, which can only come from more fund raising effort. There is much good work that can be fostered through a judicious pairing of charitable and government support. The results of this have already sown the seeds of knowledge that have turned more and more scientific attention to the problem of repairing the damaged spinal cord. Now there is a climate of guarded optimism that the Trust must nourish by continuous commitment towards its goal.

This commitment need not be confined to the small number of clinicians, scientists and patients who form the Trust's present constituency. All those reading this journal have a vital interest in improving treatment for the paralysed and many have a great capacity to help. Any such assistance is both needed and welcome in the pursuit of a desirable and permanent achievement for medical science.

Professor P. D. Wall London, UK 